\title{
Corona könnte auch andere Epidemien begünstigen
}

SARS-CoV-2 wird sich weit über 2021 hinaus auf die Reisemedizin auswirken: Fachleute rechnen mit einem deutlichen Anstieg der Masern-, Malaria- und Dengue-Fallzahlen. Bei Letzterem könnte es demnächst eine Impfung geben.

Die SARS-CoV-2-Pandemie hat sich nicht nur direkt auf uns Menschen, sondern auch auf andere Erreger dramatisch ausgewirkt:Während die aktuelle Influenzasaison weltweit quasi ausgefallen ist (in Deutschland gab es 2020/2021 bis dato gerade einmal 449 gemeldete Infektionen, im Jahr zuvor waren es bis zum selben Zeitraum 119.280 Infektionen gewesen), rechnen Experten mit einem deutlichen Anstieg etwa der hochinfektiösen Masernerkrankungen, weil Impfkampagnen auf- grund der COVID-19-Pandemie ausgefallen sind.

„Auch mückenübertragene Erkrankungen haben wieder deutlich zugenommen, nachdem Mückenkontrollprogramme im vergangenen Jahr in vielen Ländern aufgrund des Lockdowns nicht fortgeführt wurden", berichtete Professor Tomas Jelinek beim 22. Forum Reisen und Gesundheit. Daher sei auch weiter mit einem Anstieg der Malaria- und Dengue-Fallzahlen zu rechnen.
Das 22. Forum Reisen und Gesundheit fand am 12. und 13. März 2021 parallel zur Reisemesse ITB in Berlin und digital statt.

\section{Impfung gegen Dengue noch 2021?}

Gerade beim Dengue-Fieber, das auch bei deutschen Reiserückkehrern seit 2010 mit steigender Tendenz gemeldet wird, könnte künftig allerdings eine Reiseimpfung zur Verfügung stehen: Wie Dr. Eckhardt Petri von Takeda berichtete,

Hier steht eine Anzeige. 
Erstpublikation: www. aerztezeitung.de hat der Hersteller für den Dengue-Impfstoff TAK-003 kürzlich einen Antrag auf Marktzulassung bei der Europäischen Arzneimittelagentur EMA gestellt.

Die Vakzine ist gegen alle vier Dengue-Serotypen gerichtet und erreichte Petri zufolge in den zulassungsrelevanten Studien eine Wirksamkeit von $80,2 \%$ vor symptomatischer Erkrankung und von 90,4\% vor Hospitalisierung in den zwölf Monaten nach 2. Immunisierung.

Bei TAK-003 handelt es sich um einen lebend-attenuierten Impfstoff, der auf einem Backbone-Virus vom Dengue-Serotyp 2 basiert. Das Konstrukt enthält zudem drei rekombinante Dengue-Viren, die jeweils Strukturgene für Viren der Serotypen 1, 3 und 4 beinhaltet. Die Vakzine wird vermutlich mit zwei Dosen s. c. im Abstand von drei Monaten zu verimpfen sein und könnte noch in diesem Jahr in der EU zugelassen werden.

\section{Bündel von COVID-19-Vakzinen macht Reisen möglich}

Dass Reisen generell auch 2021 möglich sein wird, davon geht Reisemediziner Jelinek aus. Es stehe ein Bündel von bereits zugelassenen oder kurz vor der Zulassung stehenden COVID-19-Impfstoffen zur Verfügung, die das Reisen wieder möglich machen werden - "das Problem in Deutschland ist allerdings der Impfstoffmangel".

Am 11.03.2021 war in der EU als vierte SARS-CoV-2-Vakzine der Einzeldosis-Impfstoff As26.COV2.S des USHerstellers Johnson \& Johnson zugelassen worden.

Jelinek betonte, dass die schnelle Zulassung der COVID-19-Vakzinen keinesfalls einer mangelnden Sorgfalt geschuldet ist: „Die Phasen der klinischen Prüfung wurden nicht, wie üblich, nacheinander durchgeführt, sondern parallel - was den Prozess natürlich deutlich beschleunigt, aber auch ein deutlich höheres finanzielles Risiko für die Hersteller bedeutet."

Bei der Impfstoffherstellung liege das Risiko, noch in Phase III zu scheitern - also der letzten Phase der klinischen Prüfung - bei ganzen $50 \%$.

Autorin: Anne Bäurle

Hinweis des Verlags. Der Verlag bleibt in Hinblick auf geografische Zuordnungen und Gebietsbezeichnungen in veröffentlichten Karten und Institutsadressen neutral.

Paediatr. Paedolog. 2021 · 56:137-138 https://doi.org/10.1007/s00608-02100894-6

(c) Springer-Verlag GmbH Austria, ein Teil von Springer Nature 2021

Hier steht eine Anzeige. 